\section{\$22. Conditions for the Release of a Metallic Dust Particle from a Plasma-Facing Wall}

Ueno, M., Ohno, N. (Nagoya Univ.), Tomita, Y., Kawamura, G.

In this study the 1D plasma sheath model is applied to investigate release condition of a dust particle. A spherical dust particle resides on a vertical or horizontal flat plasma-facing wall (PFW). The magnetic field is normally applied to the PFW. The radius of the dust particle $R_{d}$ is assumed to be much smaller than the Debye length, where the dust does not affect the potential profile near the PFW. The electrostatic wall potential drop is determined by the floating condition. The velocity distribution functions of electrons and ions at the sheath entrance are assumed to be Maxwellian and Maxwellian distributions shifted by the ion sound speed, respectively. The wall potential $\phi_{w}$ is obtained as:

$$
\frac{2}{1+\operatorname{erf}\left(\sqrt{-e \phi_{w} / T_{e}}\right)} \exp \left(e \phi_{w} / T_{e}\right)=\sqrt{2 \pi \frac{m_{e}}{m_{i}}\left(Z_{i}+\frac{T_{i}}{T_{e}}\right)} .
$$

Here, the error function arises from the truncation of the electron velocity distribution function. From this condition the normalized wall potential drop - $e \phi_{w} / T_{e}$ is expressed by the temperature ration $T_{i} / T_{e}\left(=\tau_{i e}\right)$.

Both the dust particle and the PFW are negatively charged. Therefore, the normalized electrostatic force $\bar{F}_{E}$ repels the dust particle from the PFW:

$$
\bar{F}_{E}\left(\tau_{i e}\right)=\frac{F_{E}}{\pi R_{d}^{2} n_{s e} T_{e}}=\frac{4 \pi}{3}\left[\eta_{e w}\left(\tau_{i e}\right)-\eta_{i w}\left(\tau_{i e}\right)\right],
$$

where $n_{s e}$ and $T_{e}$ are the electron density and electron temperature at the sheath entrance, respectively. The contributions $\eta_{e w}$ and $\eta_{i w}$ are defined as a function of $\tau_{i e}$ :

$$
\begin{gathered}
\eta_{e w}\left(\tau_{i e}\right) \equiv \frac{1}{1+\operatorname{erf}\left(\sqrt{-e \phi_{w} / T_{e}}\right)}\left[e^{e \phi_{w} / T_{e}}-1\right. \\
\left.-\operatorname{erf}\left(\sqrt{-e \phi_{w} / T_{e}}\right)+\frac{2}{\sqrt{\pi}} e^{e \phi_{w} / T} \sqrt{-e \phi_{w} / T_{e}}\right], \\
\eta_{i w}\left(\tau_{i e}\right) \equiv \frac{4 \tau_{i e}}{\sqrt{\pi}\left[1+\operatorname{erf}\left(c_{s} / v_{t h i}\right)\right]} \\
\int_{0}^{\infty} d u u\left(u-\sqrt{u^{2}-\frac{Z_{i} e \phi_{w}}{T_{e}}}\right) \exp \left[-\left(u-\frac{c_{s}}{v_{t h i}}\right)^{2}\right],
\end{gathered}
$$

where $v_{t h i}=\sqrt{2 T_{i} / m_{i}}$.

Plasma ions are accelerated toward the PFW by the Debye sheath potential drop. Far from the dust particle, accelerated ions are absorbed by the PFW before changing their momentum. Therefore, the ion friction force due to the Coulomb scattering of plasma ions is negligibly small compared to the force due to the absorption of ions. The normalized friction force due to the absorption of plasma ions $\bar{F}_{i a b}$ is expressed as:

$$
\begin{gathered}
\bar{F}_{i a b}\left(\tau_{i e}\right)=\frac{F_{i}}{\pi R_{d}^{2} n_{s e} T_{e}}=\frac{4 \tau_{i e}}{\sqrt{\pi}}\left\{1+\operatorname{erf}\left[c_{s} / \nu_{t h i}\left(\tau_{i e}\right)\right]\right\} \\
\int_{0}^{\infty} d u u \sqrt{u^{2}-\frac{Z_{i} e \phi_{w}\left(\tau_{i e}\right) / T_{e}}{\tau_{i e}}} \exp \left\{-\left[u-c_{s} / \nu_{t h i}\left(\tau_{i e}\right)\right]^{2}\right\}
\end{gathered}
$$

Here, we apply the geometrical cross section $\pi R_{d}{ }^{2}$ as the ion absorption one for the accelerated plasma ions. The ion absorption force at $T_{i}=0$ is expressed as:

$$
\bar{F}_{i a b}\left(\tau_{i e}=0\right)=\sqrt{1-2 e \phi_{w} / T_{e}} .
$$

The normalized forces $\bar{F}_{E}$ and $\bar{F}_{i a b}$ depend on the plasma temperature ratio $\tau_{i e}$ and the normalized gravity depends on the quantity $R_{d} / n_{s e} T_{e}$. Therefore, the dust release condition is determined by two parameters, $\tau_{i e}$ and $R_{d} / n_{s e} T_{e}$.

In the case of a dust particle on a vertical wall, the release condition is determined by the balance of the electrostatic force and the ion friction force and is governed by the plasma temperature ratio $\tau_{i e}$. In Fig. 1, the relation of these forces is shown for a hydrogen plasma. The ion friction force increases almost linearly with $\tau_{i e}$. The electrostatic repelling force $\bar{F}_{E}$ is balanced by the ion friction force $\bar{F}_{i a b}$ at $\tau_{i e} \sim 0.10$. At ion temperatures lower than $0.10 T_{e}$, there is a possibility that the dust particle releases from the PFW due to the larger electrostatic force.

In the case of a dust particle on a horizontal wall, the dust release condition depends on both $\tau_{i e}$ and $R_{d} / n_{s e} T_{e}$. At $\tau_{i e}$ $=0$, the dust release condition for the hydrogen plasma is obtained as the relation $\bar{F}_{g}<\bar{F}_{E}-\bar{F}_{i a b}(=2.816-2.587=$ 0.229 ) (Fig. 1). The dust release condition is expressed as:

$$
\frac{\rho_{d, \mathrm{~g} / \mathrm{cc}} R_{d, \mu \mathrm{m}}}{n_{s e, 18} T_{e, \mathrm{eV}}}<2.80,
$$

where $\rho_{d, \mathrm{~g} / \mathrm{cc}}, R_{d, \mu \mathrm{m}}, n_{s e, 18}$ and $T_{e, \mathrm{eV}}$ are the units of $\mathrm{g} / \mathrm{cc}$ of the dust mass density, $\mu \mathrm{m}$ of the dust radius, $10^{18} \mathrm{~m}^{-3}$ of the electron density at the sheath entrance and $\mathrm{eV}$ of the electron temperature, respectively. For the case $R_{d}=1 \mu \mathrm{m}$, $n_{s e}=10^{18} \mathrm{~m}^{-3}$ and $\tau_{i e}=0$, the release condition for a tungsten and a carbon dust particle is $T_{e}>7.1$ and $0.71 \mathrm{eV}$, respectively. For the finite ion temperature, the threshold value becomes smaller than 2.80 in Eq.(7). Therefore, for the release of a dust particle the much higher electron temperatures are required.

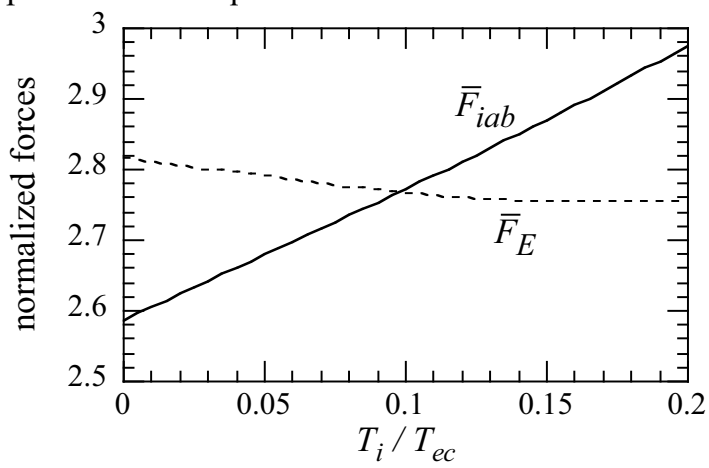

Fig. 1 Normalized ion friction force and electrostatic force on a dust particle as a function of $\tau_{i e}$. 